\title{
Protection of Indoor Air from Radioactive Gas Radon
}

\author{
Igor Shubin ${ }^{1}$, Natalya Bakaeva ${ }^{1,2^{*}}$, Alexander Kalaydo ${ }^{1}$ \\ ${ }^{1}$ Russian Academy of Architecture and Building Science Research Institute of Building Physics, \\ 127238, Moscow, Russia \\ ${ }^{2}$ Moscow State University of Civil Engineering, Yaroslavskoe shosse, 26, Moscow, 129337, Russia
}

\begin{abstract}
A modern human receives most of the radioactive annual dose in buildings from radon and its progeny, which can cause lung cancer. In such conditions, the design of radon-safe buildings is an urgent task in the context of preserving the population collective health. The paper shows the inefficiency of using the radon flux density from the soil surface as a criterion for the potential radon hazard of a construction site due to its significant temporal and spatial variability. The design of an experimental device, which makes it possible to simulate the real conditions of radon transport in soil in laboratory conditions, is described, and the results of determining the dominant radon transport mechanism in soil by means of a laboratory experiment are presented. A method for determining the required radon resistance of the floor structure is proposed on the basis of the diffusion model of stationary transport.
\end{abstract}

\section{Introduction}

It has now been established that household exposure to radon-222 and its short-lived progeny forms the majority of the individual annual exposure dose to the population of the Russian Federation and foreign countries. Radon exposure significantly exceeds the power of other radiation exposure sources of the population and is the second cause of lung cancer after smoking [1-3]. However, modern society is still insufficiently informed about the dangers of indoor radiation and fears only radiation accidents at nuclear power plants.

Radon is a noble, colorless, odorless, radioactive monatomic gas, one of the least abundant elements on Earth.This name refers to the three natural isotopes radon ${ }^{222} \mathrm{Rn}$, thoron ${ }^{220} \mathrm{Rn}$ and actinon ${ }^{219} \mathrm{Rn}$, which are continuously formed in the soil in three radioactive families from parent radium (Fig. 1). However, only the main isotope radon-222 poses a real threat to the indoor environment of buildings [4]. Its half-life is long enough (3.82 days) and it is able to migrate in the porous medium over considerable distances, reach the underground shell of buildings, overcome it and accumulate in the indoor air of the lower floor [5-6].

\footnotetext{
* Corresponding author: natbak@mail.ru
} 
The process of the unfavorable radon situation formation in a building can be conditionally divided into 3 stages: the radon formation in the soil, its transport in the geological medium and materials of the walling and its accumulation in the indoor air. The greatest difficulty is the description of the radon transport in porous media due to the large number of factors that control this process.

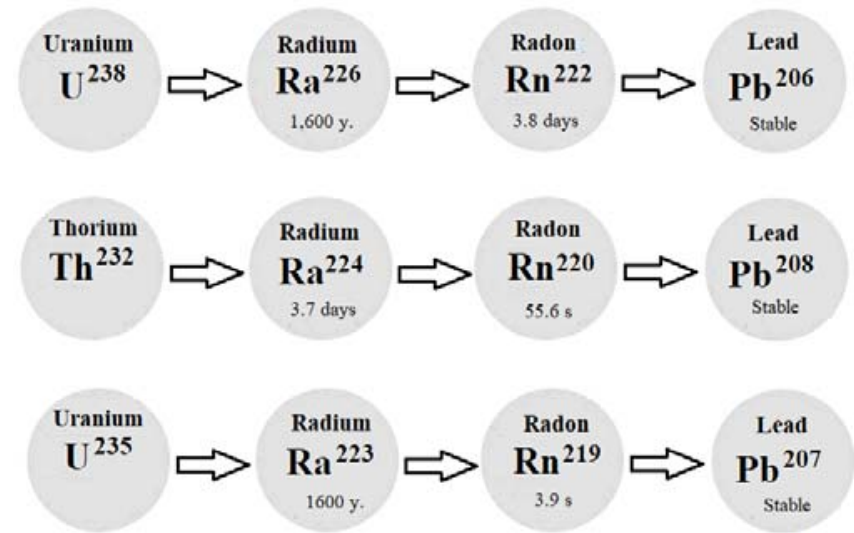

Fig. 1. Diagram of the formation and decay of radon isotopes

The rate of radon generation in the soil $G$ depends on the radium specific activity and the soil density. But not all formed in the soil radon atoms entry into the pore space and can freely move in it. Some of them get inside the neighboring soil grains and do not take part in the transport to the soil surface. The fraction of radon atoms that are capable of migration in the soil block is described by the emanation coefficient $k_{e m}$. Thus, the rate of radon generation can be written as

$$
G=\frac{C_{R a} \cdot \rho \cdot k_{e m} \cdot \lambda}{\varepsilon}
$$

where $C_{R a}$ is the radium specific activity in the soil, $\mathrm{Bq} \cdot \mathrm{kg}^{-1} ; \rho$ is the soil density, $\mathrm{kg} \cdot \mathrm{m}^{-3} ; \lambda$ is the radon decay constant, $\mathrm{s}^{-1} ; \varepsilon$ is the soil porosity.

The rate of radon generation in the soil is one of the most important values in the design of radon-safe buildings, since it determines the maximum radon activity in the soil

$$
A_{\text {max }}=\frac{G}{\lambda}=\frac{C_{R a} \cdot \rho_{z p} \cdot k_{э м}}{\varepsilon},
$$

which depends the intensity of radon entry into the indoor air.

The radon in the soil under the influence of concentration and pressure differences is transferred towards the soil surface and enters the atmosphere. About 20 years ago, at the initial stage of the radon problem study, it was believed that the value of the radon flux density from the soil surface is a reliable characteristic of the radon hazard of a construction site. This fact is reflected in the radiation safety standards, according to which the average value of the radon flux density from the soil surface $R \leq 80 \mathrm{mBq} \cdot \mathrm{m}^{-2} \mathrm{~s}^{-1}$ unambiguously indicates the radon safety of the territory [7].

However, the process of radon transfer to the soil surface strongly depends on the soil characteristics (porosity and moisture) and external conditions (temperature and pressure fields). Since many of these factors are in continuous change, the radon flux density from the soil surface is extremely variable even over short time intervals. Many researchers note its changes 5-10 times during the day, as a result of which the building site can be classified as both radon-hazardous and radon-safe. In addition, the radon flux density from the soil surface is characterized by spatial variability, often not inferior in magnitude to temporal. 
The complexity of an adequate assessment of the arithmetic mean radon flux density over the construction site was noted in the works of M. Zhukovsky and I. Yarmoshenko [89], the disadvantages of using this value as a criterion for the radon hazard of the construction site were substantiated in the P. Miklyaev investigations [10-12], and L. Gulabyants [13-15] proposed a model approach to determining the radon load to the underground buildings shell without using the radon flux density. These studies unambiguously indicate the futility of field measurements in assessing the radon hazard level of a building site and the need to introduce a new computational and experimental approach using the results of measurements of stable soil characteristics at the construction site as input parameters.

\section{Methods}

Assessment of the radon hazard of a building site is an important, but intermediate stage in the design of a radiation safe building. It determines the required radon protection capacity of the underground walling, on the basis of which the most rational floor design is justified.

The approach to the design of buildings radon protection on the basis of a model study of the radon transport in porous media was developed in the works of the Research Institute of Building Physics scientists I. Shubin, N. Bakaeva and A. Kalaydo [16-18]. Its key point was the substantiation of the dominant transport mechanism in porous media.

Radon in soil and building walling materials can be transported by diffusion and convective mechanisms. Convective transport is caused by the pressure gradient along the depth of the soil block, its value is determined from Darcy's law

$$
q_{\text {con }}=\frac{k}{\mu} \cdot \frac{\partial P}{\partial z} \cdot A
$$

where $k$ is the soil air permeability, $\mathrm{m}^{2} ; \mu$ is the air dynamic viscosity, taken in calculations equal $1,7 \cdot 10^{-5} \mathrm{~Pa} \cdot \mathrm{s}$.

Radon diffusion in porous media is due to the difference in its concentration at the outer boundaries of porous media. The diffusion flow density is determined from Fick's law

$$
q_{\text {dif }}=D \cdot \frac{\partial A}{\partial z}
$$

where $D$ is the radon diffusion coefficient, $\mathrm{m}^{2} \cdot \mathrm{s}^{-1}$.

The pressure gradient in the soil rarely exceeds $1 \mathrm{~Pa} \cdot \mathrm{m}^{-1}$ [19], but the permeabilities of soils are in the range from $10^{-10}$ to $10^{-15} \mathrm{~m}^{2}$, as a result, the density of the convective flux can vary over a very wide range. On the contrary, the diffusion flux remains practically constant, since the radon transport to the atmosphere is compensated by its formation in the soil block, and the changes in the radon concentration in indoor air are negligible compared to the its concentration in the soil. The main difference between the radon diffusion transport in the walling materials is it does not require air gaps in the underground building envelope for its flow.

Determining the dominant radon transport mechanism plays a key role in simulating the radon situation in a building. If diffusion is the only significant transport mechanism, then we can talk about the stationary character of radon transport under the influence of the radon concentration difference

$$
\Delta A=A_{\text {soil }}-A_{\text {indoor }} \approx A_{\text {max }} .
$$

If the convective transport takes place, the radon is able to enter the underground walling from much greater depths and the radon load on the floor structures under certain conditions can greatly exceed the maximum equilibrium radon concentration in the soil $A_{\max }$. 
As mentioned above, the determination of the dominant radon transport mechanism in the in-situ study is extremely difficult due to the multifactorial character of this process. At the same time, a laboratory experiment makes it possible to carry out such studies by controlling the parameters that affect the radon transport in a porous medium.

\section{Results}

In order to study the regularities of radon transfer in porous media in laboratory conditions at the Research Institute of Building Physics an experimental device was developed (Fig. 2), consisting of a polymer pipe $160 \mathrm{~mm}$ in diameter and $1,500 \mathrm{~mm}$ in height, filled with zirconium concentrate. Zirconium formed the medium for the radon transport and at the same time was its powerful source due to the high content of natural radionuclides $\left(A_{\text {eff }} \approx 4,500\right.$ $\left.\mathrm{Bq} \cdot \mathrm{kg}^{-1}\right)$. This specific activity makes it possible to obtain high radon flux densities, but at the same time it is not sufficient to classify the concentrate as a radioactive substance that requires special service.

In the lower chamber $I$ of the experimental device the inputs of the pneumatic and electrical systems were located, the temperature difference along the column of the porous medium was created by two ceramic resistors $R_{1}=R_{2}=13 \mathrm{k} \Omega$ connected in parallel, which were connected through a temperature controller. Also, in the lower part of chamber $I$ there was an inlet of a pneumatic system designed to create a pressure drop across the column of a porous medium. The pneumatic system included a low-power constant-acting compressor that supplied air through a flowmeter. To measure the pressure drop in the device three inputs were implemented at a distance of $0.7 \mathrm{~m}$ from each other, which were connected to a highsensitivity differential pressure gauge by means of brass pipes.

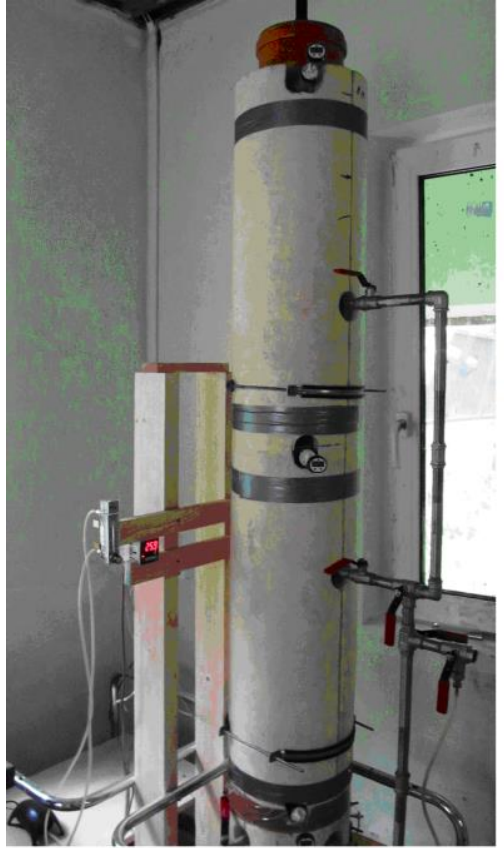

a

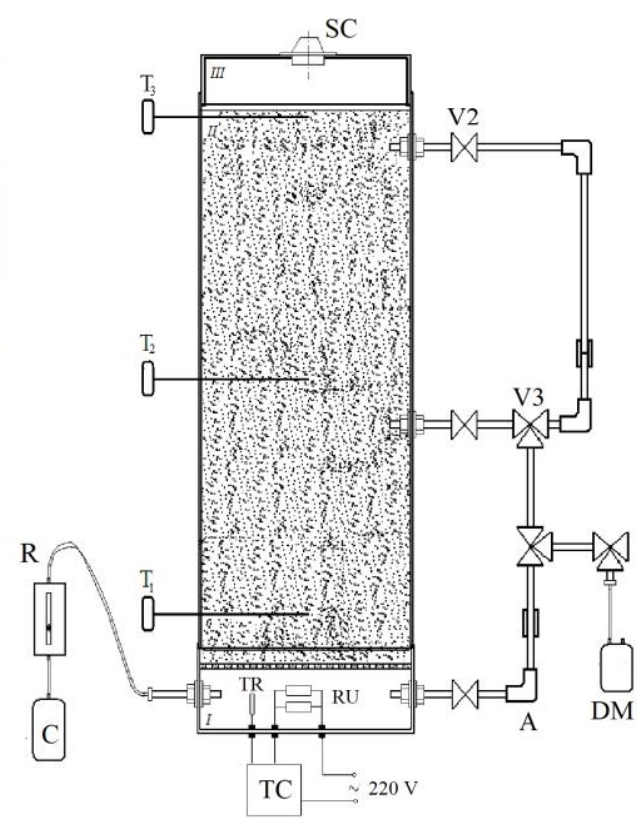

$b$

Fig. 2. The device for studying the radon transport in porous media: $a$ - appearance; $b$-scheme: SC - storage chamber; $\mathrm{R}$ - rotameter; $\mathrm{T}_{1}-\mathrm{T}_{3}$ - immersion thermometers; $\mathrm{TC}$ - thermal controller; $\mathrm{TR}$ thermal resistance; RU - resistance unit; V2 - two-way valve; V3 - three-way valve; A - $90^{\circ}$ angle adapter; $\mathrm{C}$ - compressor; $\mathrm{Co}$ - coupling; DM - differential manometer 
Initially, the radon flux density was determined under purely diffusive transport conditions in the absence of pressure and temperature drops across the zirconium column. The measurements were carried out from the moment of assembling the experimental device until the onset of radioactive equilibrium in the sealed upper chamber.

To assess the significance of the convective radon transport contribution at typical porous medium air permeability $\left(\sim 10^{-12} \mathrm{~m} 2\right)$, a temperature (from 5 to $20^{\circ} \mathrm{C}$ ) and pressure difference was alternately created. The obtained values of the radon flux density from the surface of the porous medium were compared with the results of measurements in the absence of external influences.

A statistically significant increase in the radon flux density took place at pressure drops $\Delta P \geq 50 \mathrm{~Pa}$ on a column with a height of $1.5 \mathrm{~m}$. In real conditions, the temperature-induced pressure gradient does not exceed $1-2 \mathrm{~Pa} \cdot \mathrm{m}^{-1}$, then it is possible to make an assumption about the diffusive nature of radon transport in soils with a permeability no more than $10^{-12} \mathrm{~m}^{2}$.

\section{Discussion}

The results of studying the radon transport mechanism in soils and walling materials allow us to propose the following approach to protecting the buildings indoor air from the radioactive gas radon entry. So, the equation of one-dimensional stationary diffusion transport of radon in soil can be written in the form

$$
D \cdot \frac{\partial^{2} A}{\partial z^{2}}-\lambda A+G=0
$$

with initial conditions

$$
A(0)=0 \mathrm{~Bq} \cdot \mathrm{m}^{-3}, A(\infty)=A_{\max } \mathrm{Bq} \cdot \mathrm{m}^{-3} .
$$

The solution to equation (5) has the form

$$
A(z)=A_{\max } \cdot\left(1-e^{-\frac{z}{L_{d}}}\right)=A_{\max } \cdot\left(1-e^{-z \cdot \sqrt{\frac{\lambda}{D}}}\right),
$$

where $L_{d}$ is the radon diffusion length, $\mathrm{m} ; D$ is the radon diffusion coefficient, $\mathrm{m}^{2} \cdot \mathrm{s}^{-1}$.

Table 1 shows the values of the diffusion coefficient and the diffusion length of radon

\begin{tabular}{|c|c|c|}
\hline Soil type & $\begin{array}{c}\text { Diffusion coefficient } \\
D, \mathrm{~m}^{2} \cdot \mathrm{s}^{-1}\end{array}$ & $\begin{array}{c}\text { Diffusion length } \\
L_{d}, M\end{array}$ \\
\hline $\begin{array}{l}\text { Seasonal banded clays in nature } \\
\text { conditions }\end{array}$ & $(0,65 \ldots 0,75) \cdot 10^{-6}$ & $0,56 \ldots 0,60$ \\
\hline Loams in natural conditions & $(1,0 \ldots 3,0) \cdot 10^{-6}$ & $0,69 \ldots 1,20$ \\
\hline Compacted clay sand & $2,6 \cdot 10^{-6}$ & 1,11 \\
\hline $\begin{array}{l}\text { Loam (moisture } 5.7 \% \text {, porosity } \\
0,408 \text { ) }\end{array}$ & $2,7 \cdot 10^{-6}$ & 1,13 \\
\hline $\begin{array}{l}\text { Crushed eluvial-diluvial soils in } \\
\text { natural conditions }\end{array}$ & $4,5 \cdot 10^{-6}$ & 1,46 \\
\hline $\begin{array}{l}\text { Deluvial, slightly moist soils in } \\
\text { natural conditions }\end{array}$ & $7,0 \cdot 10^{-6}$ & 1,83 \\
\hline
\end{tabular}
for the most common soil types.

Table 2. Diffusion length and diffusion coefficient of radon in various soils [20]

Substituting in (6) the depth of the foundation $z=h$ and the soil characteristics at the building base $\left(D\right.$ or $L_{d}$ ) it is possible to determine the radon concentration at the outer boundary of the horizontal underground walling $A(h)$, equal to the radon load on the floor 
structure $\Delta A$. In the future, the task is reduced to determining the required radon resistance of the floor structure.

$$
R_{\text {rad }}=\frac{\Delta A}{q_{\text {dif }}} .
$$

where $q_{\partial u \phi}$ is the limiting radon flux density through the floor structure, $\mathrm{Bq} \cdot \mathrm{m}^{-2} \cdot \mathrm{s}^{-1}$.

The $q_{\text {dif }}$ value is uniquely determined by the acceptable radon concentration in the indoor air after the building construction. The total radon resistance of the floor structure is determined by the number and physical characteristics of its layers.

At the last stage, the total radon resistance between individual layers is broken down according to the formula

$$
R_{\text {rad }}=\sum_{i=1}^{n} R_{i}=\sum_{i=1}^{n} \frac{1}{\sqrt{\lambda D_{i}}} \cdot \operatorname{sh}\left(H_{i} \sqrt{\frac{\lambda}{D_{i}}}\right),
$$

where $n$ is the number of layers in the floor structure; $H_{i}$ are the layer thicknesses, m; $D_{i}$ are the diffusion coefficients of radon in layer materials, $\mathrm{m}^{2} \cdot \mathrm{s}^{-1}$.

As practice shows, to ensure the required radon protection capacity of the underground building envelope, it is sufficient to limit ourselves to two layers with the greatest radon resistance, such as reinforced concrete and polymer film materials.

\section{Conclusions}

The presented studies allow us to conclude that the design of radon-safe buildings is possible only on the basis of reliable information about the degree of radon hazard of the building site. In this case, it is necessary to abandon the use of the radon flux density from the soil surface as a criterion for the radon hazard of the territory in favor of measuring less variable characteristics.

Since in the overwhelming majority of cases the permeability of the soil under the building does not exceed $10^{-12} \mathrm{~m}^{2}$, at determining the radon load on underground walling we can talk about the only diffusion nature of the radon transport. This assumption greatly simplifies the modeling of the process of radon accumulation in buildings without compromising the correctness of the results obtained.

\section{References}

1. Clement C 2010 ICRP Publication 115: Lung Cancer Risk from Radon and Progeny and Statement on Radon Annals of the ICRP 40 (11) 64.

2. Darby S, Hill D and Auvinen A, et al 2005 Radon in homes and risk of lung cancer: collaborative analysis of individual data from 13 European case-control studies. $\mathrm{Br}$. Med. J. 330 223-227.

3. Romanovich I, Stamat I, Kormanovskaya T, Kononenko D. et al. 2018 Natural sources of ionizing radiation: radiation doses, radiation risks, preventive measures] St. Petersburg: FBUN NIIRG im. P.V. Ramzaeva 432.

4. Jelle B 2012 Development of a model for radon concentration in indoor air Science of the Total Env. 416 343-350. 
5. Chen J, Moir D and Whyte J 2012 Canadian population risk of radon induced lung cancer: a re-assessment based on the recent cross-Canada radon survey Radiat. Prot. Dosim. 151 (2) 144-153.

6. Wang F and Ward I C 2002 Radon entry, migration and reduction in houses with cellars Building and Environment 37 pp. 1153-1165.

7. Radiation safety standards (NRB-99/2009) Ministry of Justice of Russia 2009. 225.

8. Yarmoshenko I, Zhukovsky M, Ekidin A 1999 The Modeling of radon entry in dwellings ANRI 4 17-26.

9. Vasilyev A and Zhukovsky M 2013 Determination of mechanisms and parameters which affect radon entry into a room J. Environ. Radioact 124 185-190.

10. Miklyaev P and Petrova T 2007 Mechanisms of formation of radon flux from the soil surface and approaches to assessing the radon hazard of residential areas ANRI 2 2-16

11. Miklyaev P, Petrova T, Tsapalov A and Borisov A 2012 Experience of using the isotope geochemical method to study the conditions of radon transport to the surface ANRI 1 $15-20$

12. Miklyaev P S, Petrova T B. 2018 Problems of assessment and mapping of geogenic radon potential. Analysis, forecast and management of natural risks taking into account global climate change "Georisk-2018" Materials of the X International Scientific and Practical Conference on the reduction of natural hazards and risks pp 87-92.

13. Gulabyants L 2013 Guide the radon protection design of the residential and public buildings Moscow: FEN-SCIENCE, 52.

14. Gulabyants L, Livshits M, Medvedev S 2016 The Measurement of Radon Impact on Underground Building Envelope The Academy 1 122-128.

15. Gulabyanc L, Kalaydo A and Livshic M 2017 Mathematical model of the formation of a radon environment in a building ANRI 1 (88) 41-49.

16. Shubin I, Bakaeva N, Kalaydo A, Skrynnykova A 2020 The Evaluation of RadonProtective Characteristics in Engineered and Existing Buildings with the Radon Diffusive Entry from the Soil IOP Conf. Ser.: Materials Science and Engineering $\mathbf{7 5 3}$ 032076. doi:10.1088/1757-899X/753/3/032076.

17. Shubin I, Bakaeva N, Kalaydo A 2021 Radon Safety of Designed Buildings in Russia and USA IOP Conf. Ser.: Materials Science and Engineering 1030012027. doi:10.1088/1757-899X/1030/1/012027.

18. Bakaeva N, Kalaydo A 2018 Analytical model for calculation the radon-protective characteristics of underground walling IOP Conf. Ser.: Materials Science and Engineering 456 012102. doi:10.1088/1757-899X/456/1/012102.

19. Andersen C 2001 Numerical modelling of radon-222 entry into housesThe Science of the Total Environment 272 33-42.

20. Andersen C E 1992 Entry of Soil Gas and Radon into Houses, Riso Nat. Lab., Roskilde, Riso-R-623. 